\title{
Non-linear heat and mass transfer in a thermal radiated MHD flow of a power-law nanofluid over a rotating disk
}

\author{
Nabil T. EL-Dabe ${ }^{1} \cdot$ Hazim A. Attia ${ }^{2} \cdot$ Mohamed A. I. Essawy $^{3,4} \cdot$ Ibrahim H. Abd-elmaksoud $^{2} \cdot$ Ahmed A. Ramadan $^{4}$. \\ Alaa H. Abdel-Hamid ${ }^{4}$
}

(c) Springer Nature Switzerland AG 2019

\begin{abstract}
The steady MHD flow of a power law nanofluid due to a uniform rotation of an infinite disk is studied with heat and mass transfer. The viscous dissipation has been comprised in the energy equation. The governing PDEs are reduced to a set of ODEs; using the generalized Von Karman similarity transformations; for which finite difference numerical scheme is implemented along with the associated boundary conditions. The non-Newtonian fluid characteristics affect the fluid velocity, temperature and concentration of suspended nanoparticles. The significant effects of thermal radiation, Brownian motion and thermophoresis diffusion are involved. The skin friction coefficients in addition to the heat and mass transfer rates are defined and calculated considering the variation of all flow parameters. The present results are verified and compared with literature.
\end{abstract}

Keywords MHD flow · Power-law fluid · Nanofluids · Rotating disk · Heat and mass transfer $\cdot$ Numerical solution

\begin{tabular}{|c|c|c|c|}
\hline \multicolumn{2}{|c|}{ List of symbols } & $n$ & The power-law index \\
\hline$(r, \varphi, z)$ & Cylindrical coordinates & $\mu_{o}$ & Consistence coefficient \\
\hline$(u, v, w)$ & Radial, azimuthal and vertical velocity compo- & $\mu$ & Coefficient of viscosity \\
\hline & nents; respectively & $m$ & Magnetic parameter \\
\hline$(F, G, H)$ & Non-dimensional velocity components & $\rho_{F}$ & Fluid density \\
\hline$\partial p / \partial r$ & Pressure gradient & $c$ & Specific heat capacity of the fluid \\
\hline$p_{\infty}$ & Pressure of the ambient fluid & $k$ & Thermal conductivity of the fluid \\
\hline$u$ & Kinematic viscosity of the fluid & $B_{o}$ & Uniform magnetic field \\
\hline$\zeta$ & Non-dimensional distance & $\sigma$ & Electric conductivity of the fluid \\
\hline$\omega$ & Angular velocity of the disk & $\operatorname{Pr}$ & Prandtl number \\
\hline$T$ & The temperature of the fluid & Ec & Eckert number \\
\hline$T_{w}, T_{\infty}$ & $\begin{array}{l}\text { The temperatures of the disk and ambient } \\
\text { fluid; respectively }\end{array}$ & $\begin{array}{l}D_{t} \\
D_{b}\end{array}$ & $\begin{array}{l}\text { Thermophoretic diffusion coefficient } \\
\text { Brownian motion coefficient }\end{array}$ \\
\hline$C$ & Nanoparticles concentration & $\mathrm{Nb}$ & Brownian motion parameter \\
\hline$C_{w} C_{\infty}$ & $\begin{array}{l}\text { Nanoparticles concentration of the disk and } \\
\text { ambient fluid; respectively }\end{array}$ & $\begin{array}{l}N t \\
\text { Le }\end{array}$ & $\begin{array}{l}\text { Thermophoretic parameter } \\
\text { Lewis number }\end{array}$ \\
\hline$\theta$ & Dimensionless temperature & $R_{d}$ & Radiation parameter \\
\hline$\phi$ & Dimensionless nanoparticles concentration & $q_{r}$ & Radiative heat flux \\
\hline
\end{tabular}

$\triangle$ Mohamed A. I. Essawy, mohamed.essawy@hti.edu.eg| 'Department of Mathematics, Faculty of Education, Ain Shams University, Roxy, Heliopolis, Cairo, Egypt. ${ }^{2}$ Department of Engineering Mathematics and Physics, Faculty of Engineering, Fayoum University, Fayoum 63415 , Egypt. ${ }^{3}$ Higher Technological Institute (HTI), 3rd Zone, 7th Section, 6th of October City, P.O. Box No. 4, Giza, Egypt. ${ }^{4}$ Mathematics Department, Faculty of Science, Beni-Suef University, Beni Suef 62511, Egypt. 


$\begin{array}{ll}C_{f_{t}} & \text { Tangential skin friction coefficient } \\ C_{f_{r}} & \text { Radial skin friction coefficient } \\ q_{w} & \text { Heat flux } \\ q_{m} & \text { Mass flux } \\ N u_{r} & \text { The local Nusselt number } \\ S h_{r} & \text { The local Sherwood number } \\ R e_{r} & \text { The rotational Reynolds number }\end{array}$

\section{Introduction}

Rotating disk flows are of both theoretical and practical value. The boundary layer induced by a rotating disk is of great scientific importance owing to its relevance to applications in many areas such as rotating machinery, computer storage devices, viscometry, turbo-machinery, lubrication, oceanography, crystal growth processes, and chemical vapor deposition reactor [1]. The problem of the motion of a fluid due to the rotation of an infinitely extended disk was firstly illustrated by von Karman [2], who introduced a set of generalized similarity transformations to reduce the governing PDEs to ODEs.

Many authors studied the heat transfer behavior from a rotating disk in different ways [3-5]. Batista [6] succeeded in finding a closed form for the velocity components regarding the fluid flow between two uniformly co-rotating disks. Also, explicit solutions have been presented for generalized non-Newtonian fluids at different conditions with both mechanical and biological applications [7-9]. The flow of non-Newtonian power-law fluids considering the influence of a magnetic field has been studied [10-12] using the extensions of Karman analysis which discussed in $[13,14]$. MHD flows regarding power-law fluids over a rotating disk are of great impact because of the absence of the magnetic force field outside the viscous boundary layer, which means that the fluid flow only affected inside the boundary layer. Applying an external uniform magnetic field on a power-law fluid flow over a rotating disk was proved to serve in the process of flow control [15-17].

The steady flow of a nanofluid due to a rotating disk was studied by Bachok et al. [18]. The thermal radiation effect on the motion of an electrically conducting fluid over an infinite rotating porous disk was studied with heat and mass transfer [19]. Ming et al. [20] gave extreme illustrations to the steady flow and heat transfer of an incompressible viscous fluid of a power-law type over a rotating infinite disk. They assumed that the thermal conductivity obeys the same nonlinear formula as the definition of the viscosity function. Osalusi [21] provided a continuum of the fluid motion over a rotating disk considering the Reiner-Rivlin model. Andersson et al. [22] demonstrated the characteristics of the power-law fluid flow over a rotating disk by introducing the boundary layer approximations and extending the power-law index in the range of (1.5, 2). Later, Andersson and de Korte [15] expanded their research work to account for the MHD flow; they obtained asymptotic solutions and solved numerically for magnetic parameter values up to 4.0. They concluded that imposing the magnetic field is more effective for shear-thinning than for shear-thickening fluids where a distinctive behavior has been obtained compared with the non-magnetic case.

Nanofluids provide an important class of fluids because of their enormous energetic applications. This kind of fluids composes of a base liquid with suspended nanoparticles. The fluid thermal conductivity is enhanced because of the addition of small amount of nanoparticles according to the experimental verification made by Choi [23]. Buongiorno [24] worked out his famous mathematical model that addresses the flow of nanofluids along with the incorporation of both the Brownian motion and thermophoretic diffusion of nanoparticles. Nanofluids aroused a great interest because of enhancing the thermal conductivity of the base fluid which is necessary for several applications; especially in nuclear reactors [25-27].

Bachok et al. [18] interpreted the nanofluid flow and heat transfer characteristics because of the rotation of an infinitely extended porous disk. The steady magnetohydrodynamic flow of a nanofluid due to a rotating porous disk has been richly discussed considering the entropy generation phenomenon [28]. This simulation proved the high impact of using a magnetic rotating disk in novel nuclear space propulsion engines in addition to its several applications in heat transfer enhancement. Turkyilmazoglu [29] illustrated the flow and heat transfer of many waterbased nanofluids over a rotating disk. The phenomenon of nanoparticles precipitation; accompanying to the arising motion of power-law nanofluids due to a rotating disk has been studied numerically using the Homotopy analysis method (HAM) [30]. The obtained solutions agreed with the experimental results; which reflects the importance of such mathematical formulations. Mustafa et al. [31] proposed a numerical study of a two-phase Bödewadt nanofluid flow with heat transfer over a stationary stretching disk. Later, many authors [32-35] have presented extensive research work regarding the flow of nanofluids between two rotating disks under different physical assumptions. The usage of such nanofluids enhancing the heat transfer performance and leads to many updatable energetic applications [36].

The present work ventilates the effectiveness of thermal radiation on the nonlinear heat and mass transfer across a steady MHD flow of a power-law nanofluid over a rotating disk; as a continuation of the problems discussed previously in $[15,22]$. The governing nonlinear PDEs of fluid flow, temperature and nanoparticles concentration in the prescribed boundary layer are solved numerically using 
finite differences. The effects of characteristics of nonNewtonian power-law fluid have been accentuated and a full parametric study has been conducted.

\section{Physical model and governing equations}

This problem considers the arising steady motion of a fluid due to the rotating behavior of an insulated infinite disk about the $z$-axis with angular velocity $\omega$. The cylindrical polar coordinates $(r, \varphi, z)$ are used in modeling this phenomenon as presented in Fig. 1. The disk has been positioned in the plane $z=0$. The utilized non-Newtonian power law nanofluid occupies the space $z>0$. The pressure gradient in the $z$-direction vanishes $(\partial p / \partial z=0)$ according to the boundary layer derivation represented by Andersson and de Korte [15]. In addition, the similarity transformations of Karman implied $(\partial p / \partial r=0)$, which provides a constant pressure inside the boundary layer. The disk is maintained at a constant temperature $T_{w}$, while, the fluid out of the boundary layer is kept at a uniform ambient temperature $T_{\infty}$. The concentration of the nanoparticles at the disk is set to a constant value $C_{w}$ differs from that far from the disk $C_{\infty}$. This physical geometry can

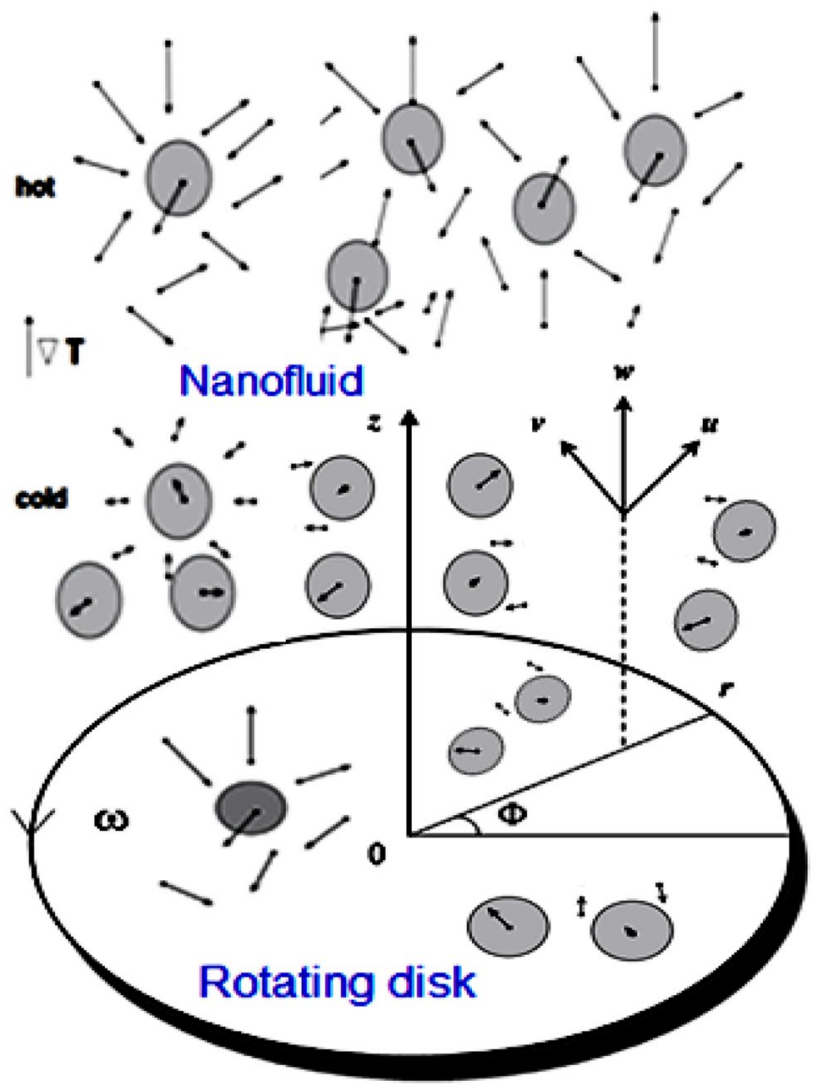

be found in many realistic situations such as rotating and turbo-machinery.

The governing PDEs of continuity, momentum, energy and concentration are given; respectively, as follows:

$\frac{\partial u}{\partial r}+\frac{u}{r}+\frac{\partial w}{\partial z}=0$

$\rho\left(u \frac{\partial u}{\partial r}+w \frac{\partial u}{\partial z}-\frac{v^{2}}{r}\right)=-\frac{\partial p}{\partial r}+\frac{\partial}{\partial z}\left(\mu \frac{\partial u}{\partial z}\right)-\sigma B_{o}^{2} u$

$\rho\left(u \frac{\partial v}{\partial r}+w \frac{\partial v}{\partial z}+\frac{u v}{r}\right)=\frac{\partial}{\partial z}\left(\mu \frac{\partial v}{\partial z}\right)-\sigma B_{o}^{2} v$

$u \frac{\partial T}{\partial r}+w \frac{\partial T}{\partial z}=\frac{k}{\rho c} \frac{\partial^{2} T}{\partial z^{2}}+\frac{\mu}{\rho c}\left[\left(\frac{\partial u}{\partial z}\right)^{2}+\left(\frac{\partial v}{\partial z}\right)^{2}\right]$

$+R\left[\frac{D_{t}}{T_{w}}\left(\frac{\partial T}{\partial z}\right)^{2}+D_{B}\left(\frac{\partial T}{\partial z} \frac{\partial C}{\partial z}\right)\right]+\frac{\sigma B_{o}^{2}}{\rho c}\left(u^{2}+v^{2}\right)-\frac{1}{\rho c} \frac{\partial q_{r}}{\partial z}$

$u \frac{\partial C}{\partial r}+w \frac{\partial C}{\partial z}=D_{B}\left(\frac{\partial^{2} C}{\partial z^{2}}\right)+\frac{D_{t}}{T_{w}}\left(\frac{\partial^{2} T}{\partial z^{2}}\right)$

The non-Newtonian fluid considered in the present work obeys the power law model, where, the viscosity has been assumed to depend on the velocity gradients as follows [15]:

$\mu=\mu_{o}\left[\left(\frac{\partial u}{\partial z}\right)^{2}+\left(\frac{\partial v}{\partial z}\right)^{2}\right]^{(n-1) / 2}$

In the last term of the right hand side of Eq. (4), $q_{r}$ accounts for the radiated heat flux [37],

$q_{r}=-\frac{4 \sigma^{*}}{3 k^{*}} \frac{\partial T^{4}}{\partial z}$

where, $\sigma^{*}$ is the Stefan-Boltzmann constant and $k^{*}$ is the mean absorption coefficient. The term $T^{4}$ is expressed as a linear Taylor expansion of temperature about $T_{\infty}$ with neglecting the second and higher order terms,

$T^{4} \cong 4 T_{\infty}^{3} T-3 T_{\infty}^{4}$

The boundary conditions are given by:

$u=0, v=r \omega, w=0, T=T_{w}$ and $C=C_{w}$ at $z=0$

$u \rightarrow 0, v \rightarrow 0, T \rightarrow T_{\infty}$ and $C \rightarrow C_{\infty}$ as $z \rightarrow \infty$.

The following Von Karman generalized transformations are modified in order to fit the present power law flow problem $[13,15]$ :

Fig. 1 Rotating disk flow configuration 
$\left\{\begin{array}{l}u=r \omega F(\zeta), \quad v=r \omega G(\zeta), \quad w=\left(\frac{\omega^{1-2 n}}{\mu_{g} / \rho}\right)^{-1 /(1+n)} r^{(n-1) /(n+1)} H(\zeta), \\ p-p_{\infty}=-\rho \omega v P(\zeta), \quad \zeta=z\left(\frac{\omega^{2-n}}{\mu_{o} / \rho}\right)^{1(1+n)} r^{(1-n) /(n+1)}, \\ \theta=\left(T(\zeta)-T_{\infty}\right) /\left(T_{w}-T_{\infty}\right), \quad \phi=\left(C(\zeta)-C_{\infty}\right) /\left(C_{w}-C_{\infty}\right) .\end{array}\right.$

With the definitions illustrated in (6) and (11); the Eqs. (1-5) are transformed to Eqs. (12-16):

$H^{\prime}+2 F+\left(\frac{1-n}{1+n}\right) \zeta F^{\prime}=0$

$F^{2}-G^{2}+\left(H+\left(\frac{1-n}{1+n}\right) \zeta F\right) F^{\prime}=\left(\left(\left(F^{\prime}\right)^{2}+\left(G^{\prime}\right)^{2}\right)^{(n-1) / 2} F^{\prime}\right)^{\prime}-m F$

$2 F G+\left(H+\left(\frac{1-n}{1+n}\right) \zeta F\right) G^{\prime}=\left(\left[\left(F^{\prime}\right)^{2}+\left(G^{\prime}\right)^{2}\right]^{(n-1) / 2} G^{\prime}\right)^{\prime}-m G$

$\left[\left(\frac{1-n}{1+n}\right) \eta F+H\right] \theta^{\prime}=\frac{1}{\operatorname{Pr}}\left[\left(1+\frac{4}{3 R_{d}}\right) \theta^{\prime \prime}+N_{t} \theta^{\prime 2}+N_{b} \theta^{\prime} \phi^{\prime}\right]$

$$
+E C\left[\left(F^{\prime 2}+G^{\prime 2}\right)^{(n+1) / 2}+m\left(F^{2}+G^{2}\right)\right]
$$

$\left[\left(\frac{1-n}{1+n}\right) \eta F+H\right] \phi^{\prime}=\frac{1}{\text { Le } \operatorname{Pr}}\left(\phi^{\prime \prime}+\frac{N_{t}}{N_{b}} \theta^{\prime \prime}\right)$

where, the differentiation is with respect to the variable $\zeta$.

The dimensionless boundary conditions are expressed as:

$F=0, G=1, H=0, \theta=1$ and $\phi=1$ at $\zeta=0$

$F \rightarrow 0, G \rightarrow 0, \theta \rightarrow 0$ and $\phi \rightarrow 0$ as $\zeta \rightarrow \infty$

The parameters that govern the fluid flow are defined in the following manner: $\left\{\begin{array}{l}\tau_{t}=\left[\mu\left(\frac{\partial v}{\partial z}+\frac{1}{r} \frac{\partial w}{\partial \varphi}\right)\right]_{z=0^{\prime}} \\ \tau_{r}=\left[\mu\left(\frac{\partial u}{\partial z}+\frac{\partial w}{\partial \varphi}\right)\right]_{z=0^{\prime}} \\ q_{w}=-\left[\left(\frac{16 \sigma^{*} T_{\infty}^{3}}{3 k^{*}}+k\right)\left(\frac{\partial T}{\partial z}\right)\right]_{z=0^{\prime}} \\ q_{m}=-D_{b}\left(\frac{\partial C}{\partial z}\right)_{z=0}\end{array}\right.$

The quantities $C_{f_{t}}, C_{f_{r}}, N u_{r}$ and $S h_{r}$ are given in their dimensionless forms as below:

$\left\{\begin{array}{l}\operatorname{Re}_{r}^{\frac{1}{1+n}} C_{f_{t}}=\left[F^{\prime 2}(0)+G^{\prime 2}(0)\right]^{\frac{n-1}{2}} G^{\prime}(0), \\ \operatorname{Re}_{r}^{\frac{1}{1+n}} C_{f_{r}}=\left[F^{\prime 2}(0)+G^{\prime 2}(0)\right]^{\frac{n-1}{2}} F^{\prime}(0), \\ \operatorname{Re}_{r}^{\frac{-1}{1+n}} N u_{r}=-\left[1+\frac{4}{3 R_{d}}\right] \theta^{\prime}(0), \\ \operatorname{Re}_{r}^{\frac{-1}{1+n}} S h_{r}=-\phi^{\prime}(0) .\end{array}\right.$

where, $\operatorname{Re}_{r}=\frac{r^{2} \omega^{2-n}}{\mu_{o} / \rho}$ is the rotational Reynolds number.

\section{Numerical solution}

Equations (12-16) are solved numerically using finite differences [39] under the boundary conditions given by Eqs. (17) and (18) to determine the velocity, temperature and nanoparticles concentration distributions for different values of the governing parameters $n, m, R_{d}, N_{t}$ and $N_{b}$ with various values of $\mathrm{Pr}, E C$ and Le numbers. The Crank-Nicolson implicit method [40] is applied.

The variables $D=-0.5(d F / d \zeta), E=-0.5(d G / d \zeta)$, $M=d \theta / d \zeta$ and $N=d \phi / d \zeta$ have been defined to reduce

$$
\left\{\begin{array}{lll}
m=\frac{\sigma B_{0}^{2}}{\rho \omega}, \quad \operatorname{Pr}=\mu_{0}^{2 /(n+1)} C_{p}\left(\omega^{3} r^{2} \rho\right)^{\frac{n-1}{n+1}} / k, & E C=\omega^{2} r^{2} / c_{p}\left(T_{w}-T_{\infty}\right), \quad L e=\frac{k}{\rho c D_{B}}, \\
N_{B}=\frac{R D_{B} \rho c\left(C_{w}-C_{\infty}\right)}{k}, \quad N_{t}=\frac{R D_{t} \rho c\left(T_{w}-T_{\infty}\right)}{T_{w} k}, & R_{d}=\frac{k k^{*}}{4 \sigma^{*} T_{\infty}^{3}} .
\end{array}\right.
$$

Below, One can find the definitions of the interesting physical quantities $C_{f_{t}}, C_{f_{r}}, N u_{r}$ and $S h_{r}$ [38]: the second order differential Eqs. (12-16) to first order ones as follows;

$C_{f_{t}}=\frac{\tau_{t}}{\rho r^{2} \omega^{2}}, \quad C_{f_{r}}=\frac{\tau_{r}}{\rho r^{2} \omega^{2}}, \quad N u_{r}=\frac{r q_{w}}{k\left(T_{w}-T_{\infty}\right)}, \quad S h_{r}=\frac{r q_{m}}{D_{b}\left(C_{w}-C_{\infty}\right)}$

where, the tangential and radial skin frictions $\tau_{\mathrm{t}}$ and $\tau_{\mathrm{r}}$; as well as the heat and mass fluxes $q_{w}$ and $q_{m}$ are expressed, respectively, as follows:

$$
\frac{d H}{d \zeta}+2 F-2\left(\frac{1-n}{1+n}\right) \zeta D=0
$$

$$
F^{2}-G^{2}-2\left(H+\left(\frac{1-n}{1+n}\right) \zeta F\right) D=-2^{n}\left(\left(D^{2}+E^{2}\right)^{(n-1) / 2} D\right)^{\prime}-m F
$$


$2 F G-2\left(H+\left(\frac{1-n}{1+n}\right) \zeta F\right) E=-2^{n}\left(\left(D^{2}+E^{2}\right)^{(n-1) / 2} E\right)^{\prime}-m G$

$\left[H+\left(\frac{1-n}{1+n}\right) \zeta F\right] M=\frac{1}{\operatorname{Pr}}\left[\left(1+\frac{4}{3 R_{d}}\right) M^{\prime}+N_{t} M^{2}+N_{b} M N\right]$ $+E C\left[2^{n+1}\left(D^{2}+E^{2}\right)^{(n+1) / 2}+m\left(F^{2}+G^{2}\right)\right]$

$\left[H+\left(\frac{1-n}{1+n}\right) \zeta F\right] N=\frac{1}{L e \operatorname{Pr}}\left(N^{\prime}+\frac{N_{t}}{N_{b}} M^{\prime}\right)$
The finite difference scheme is implemented by writing Eqs. (23-27) at the mid-point of the computational cell and then replacing the difference terms by their second order central difference approximation in $\zeta$ direction. A quasi-linearization technique is firstly applied to replace the non-linear terms at a linear stage, with the corrections incorporated in subsequent iterative steps until convergence. Finally, the resulting block tri-diagonal system is solved using the generalized Thomas-algorithm [39-41].

The finite difference representations for the resulting first order differential Eqs. (23-27) take the form:

$\frac{H_{i+1}-H_{i}}{\Delta \zeta}+2\left(\frac{F_{i+1}+F_{i}}{2}\right)-2\left(\frac{1-n}{1+n}\right)\left(\frac{\zeta_{i+1}+\zeta_{i}}{2}\right)\left(\frac{D_{i+1}+D_{i}}{2}\right)=0$

$$
\begin{aligned}
& \frac{F_{i+1} \bar{F}_{i+1}+F_{i} \bar{F}_{i}}{2}-\frac{G_{i+1} \bar{G}_{i+1}+G_{i} \bar{G}_{i}}{2}-\frac{H_{i} \bar{D}_{i}+\bar{H}_{i} D_{i}}{2}-\frac{H_{i+1} \bar{D}_{i+1}+\bar{H}_{i+1} D_{i+1}}{2} \\
& -\frac{1}{2}\left(\frac{1-n}{1+n}\right)\left[\zeta_{i}\left(F_{i} \bar{D}_{i}+\bar{F}_{i} D_{i}\right)+\zeta_{i+1}\left(F_{i+1} \bar{D}_{i+1}+\bar{F}_{i+1} D_{i+1}\right)\right] \\
& \quad+\frac{m}{2}\left(F_{i}+F_{i+1}\right)+\frac{2^{n}}{\Delta \zeta}\left[\left(\bar{D}_{i+1}^{2}+\bar{E}_{i+1}^{2}\right)^{(n-1) / 2} D_{i+1}-\left(\bar{D}_{i}^{2}+\bar{E}_{i}^{2}\right)^{(n-1) / 2} D_{i}\right]=0
\end{aligned}
$$

$$
\begin{aligned}
& \frac{G_{i+1} \bar{F}_{i+1}+\bar{G}_{i+1} F_{i+1}}{2}+\frac{G_{i} \bar{F}_{i}+\bar{G}_{i} F_{i}}{2}-\frac{H_{i} \bar{E}_{i}+\bar{H}_{i} E_{i}}{2}-\frac{H_{i+1} \bar{E}_{i+1}+\bar{H}_{i+1} E_{i+1}}{2} \\
& \quad-\frac{1}{2}\left(\frac{1-n}{1+n}\right)\left[\zeta_{i}\left(F_{i} \bar{E}_{i}+\bar{F}_{i} E_{i}\right)+\zeta_{i+1}\left(F_{i+1} \bar{E}_{i+1}+\bar{F}_{i+1} E_{i+1}\right)\right] \\
& \quad+\frac{m}{2}\left(G_{i}+G_{i+1}\right)+\frac{2^{n}}{\Delta \zeta}\left[\left(\bar{D}_{i+1}^{2}+\bar{E}_{i+1}^{2}\right)^{(n-1) / 2} E_{i+1}-\left(\bar{D}_{i}^{2}+\bar{E}_{i}^{2}\right)^{(n-1) / 2} E_{i}\right]=0
\end{aligned}
$$

$$
\begin{aligned}
& \frac{H_{i} \bar{M}_{i}+\bar{H}_{i} M_{i}}{4}+\frac{H_{i+1} \bar{M}_{i+1}+\bar{H}_{i+1} M_{i+1}}{4} \\
& \quad+\frac{1}{4}\left(\frac{1-n}{1+n}\right)\left[\zeta_{i}\left(F_{i} \bar{M}_{i}+\bar{F}_{i} M_{i}\right)+\zeta_{i+1}\left(F_{i+1} \bar{M}_{i+1}+\bar{F}_{i+1} M_{i+1}\right)\right] \\
& \quad-2^{(n+1) / 2} E c\left[\left(D_{i+1} \bar{D}_{i+1}+D_{i} \bar{D}_{i}\right)+\left(E_{i+1} \bar{E}_{i+1}+E_{i} \bar{E}_{i}\right)\right]^{(n+1) / 2} \\
& \quad-m E c\left(\frac{F_{i+1} \bar{F}_{i+1}+F_{i} \bar{F}_{i}}{2}+\frac{G_{i+1} \bar{G}_{i+1}+G_{i} \bar{G}_{i}}{2}\right) \\
& \quad-\frac{1}{\operatorname{Pr}}\left[\left(1+\frac{4}{3 R_{d}}\right)\left(\frac{M_{i+1}-M_{i}}{\Delta \zeta}\right)+N_{t}\left(\frac{M_{i+1} \bar{M}_{i+1}+M_{i} \bar{M}_{i}}{2}\right)\right]=0
\end{aligned}
$$




$$
\begin{aligned}
& \frac{H_{i} \bar{N}_{i}+\bar{H}_{i} N_{i}}{4}+\frac{H_{i+1} \bar{N}_{i+1}+\bar{H}_{i+1} N_{i+1}}{4} \\
& \quad+\frac{1}{4}\left(\frac{1-n}{1+n}\right)\left[\zeta_{i}\left(F_{i} \bar{N}_{i}+\bar{F}_{i} N_{i}\right)+\zeta_{i+1}\left(F_{i+1} \bar{N}_{i+1}+\bar{F}_{i+1} N_{i+1}\right)\right] \\
& \quad-\frac{1}{\text { Le } \operatorname{Pr}}\left[\left(\frac{N_{i+1}-N_{i}}{\Delta \zeta}\right)+\frac{N_{t}}{N_{b}}\left(\frac{M_{i+1}-M_{i}}{\Delta \zeta}\right)\right]=0
\end{aligned}
$$

The bars in the above equations refer to the previous iteration.

The computational domain $0<\zeta<\zeta_{\infty}$ can be divided into intervals of 0.001 step size each. The independence of the results from the length of the finite domain and the grid density was ensured and successfully checked by various trial and error numerical experimentations. The value $\zeta_{\infty}=20$ is adequate for all the ranges of the studied parameters. The scheme convergence is satisfied when the variables $H, F, G, D, E, \theta, \phi, M$ and $N$; have an absolute difference of $10^{-6}$ for the last two approximations for all values of $\zeta$ in the specified interval $0<\zeta<\zeta_{\infty}$. These results are found to be reduced to those given in $[14,15,20,22]$ considering a clear fluid with different flow modes; which, assures the solutions accuracy and correctness.

\section{Results and discussion}

Figures 2, 3 and 4 show that the augmentation of the magnetic parameter $m$ results in reducing the radial, tangential and axial velocities $F, G$ and $H$; respectively, where the movement of the rotating disk axially draws the surroundings toward the surface to compensate the radial outflow. Also, it is extremely obvious that the boundary layer thickness becomes thinner with increasing the power-law index $n$. The inclusion of the magnetic force field provides the same influence of velocity reduction considering different values of $n$ expressed for shear-thinning fluids $(n<1)$, Newtonian fluids $(n=1)$ and shear-thickening fluids $(n>1)$. It is worthwhile to infer that raising $m$ causes the boundary layer to be thinner; and that the variations of the velocities $F, G$ and $H$ with $m$ is more pronounced in case of non-Newtonian shear-thinning fluids. Moreover, Fig. 5 indicates that the values of the temperature $\theta(\zeta)$ and the nanoparticles concentration $\phi(\zeta)$ increase with boosting $m$ for different $n$ values. This influence is due to the presence of Lorentz force caused by the acting magnetic field that decelerates the flow around the disk.

Figure 6 exhibits a raise in the temperature profiles; while, a depression is obtained in the concentration profiles with increasing $E c$ for different kinds of fluids. This illustrates the fact that when the friction increases due to fluid viscosity, a large amount of heat is obtained where the viscous dissipation provides an important internal heat source because of the viscous stresses action; and hence, the nanofluid temperature increases. Figure 7 displays the influence of the thermal radiation parameter $R_{d}$ on both $\theta(\zeta)$ and $\phi(\zeta)$ for different $n$. Increasing $R_{d}$ elevates the behavior of $\phi(\zeta)$ but, decreases $\theta(\zeta)$ and the thickness of the thermal boundary layer due to the reduction of energy transport into fluid.

Figure 8 accentuates the reduction behavior of both $\theta(\zeta)$ and $\phi(\zeta)$ with increasing $\operatorname{Pr}$; which is physically verified due to the dependence of $\operatorname{Pr}$ on the ratio of the fluid kinematic viscosity to thermal diffusivity. In this study, the values of Pr have been chosen according to the categories; $(\operatorname{Pr} \ll 1)$ for liquid metals which have high thermal conductivity but low viscosity and $(\operatorname{Pr} \gg 1)$ for high-viscosity oils. It should be mentioned that the specific used values $\operatorname{Pr}=0.72,1.0$ and 7.0 correspond to air, electrolyte solution, and water; respectively. Figure 9 elucidates that increasing Le; increases the Newtonian fluid temperature, while, a lessening in the temperature values is recognized for non-Newtonian fluids $(n \neq 1)$. Nevertheless, a diminishment attitude of $\phi(\zeta)$ is obtained due to the increase of $L e$. This may be attributed to the physical definition of $L e$ as the ratio of thermal diffusivity to nanoparticle mass diffusivity; where it is used to characterize the heat and mass transfer through nanofluids flows.

Figure 10 clarifies that the distributions of $\theta(\zeta)$ and $\phi(\zeta)$ put up with increasing the thermophoretic parameter $N_{t}$ and that the influence of thermophoresis phenomenon is the same for different values of $n$. Physically; enhancing the thermophoretic effect results in a larger mass flux due to temperature gradient which in turn raises the concentration. This mechanism therefore, assists the diffusion of the nanoparticles and elevates the concentration profile. On the other hand, Fig. 11 is prepared to present the effect of the Brownian motion on both $\theta(\zeta)$ and $\phi(\zeta)$. The temperature of the fluid decreases with increasing $N_{b}$ for Newtonian fluids; while, an elevation in $\theta(\zeta)$ profiles is obtained with increasing $N_{b}$ regarding the class of nonNewtonian fluids $(n \neq 1)$. Furthermore, $\phi(\zeta)$ decreases with increasing $N_{b}$ for all $n$ values. This reflects the great impact of following up the influence of the Brownian motion of the particles at nanoscale level; which highly affects the thermal behaviors of the surrounding liquids by transporting energy directly by nanoparticles. The parameters $N_{b}$ and $N_{t}$ may vary in $(0, \infty)$; however, the distinctive profiles can be obtained in the range $(0,2)[42]$.

Table 1 provides a comparison between the present values of the flow characteristics $F^{\prime}(0),-G^{\prime}(0)$ and $-H(\infty)$ with those obtained in $[15,43]$. These numerical results have been calculated for the particular case of a Newtonian fluid $(n=1)$. The wall gradient $F^{\prime}(0)$ and the axial inflow $-H(\infty)$ showed a reduction behavior with respect 
Fig. 2 Variation of the radial velocity $F$ with different values of $m$ and $n$
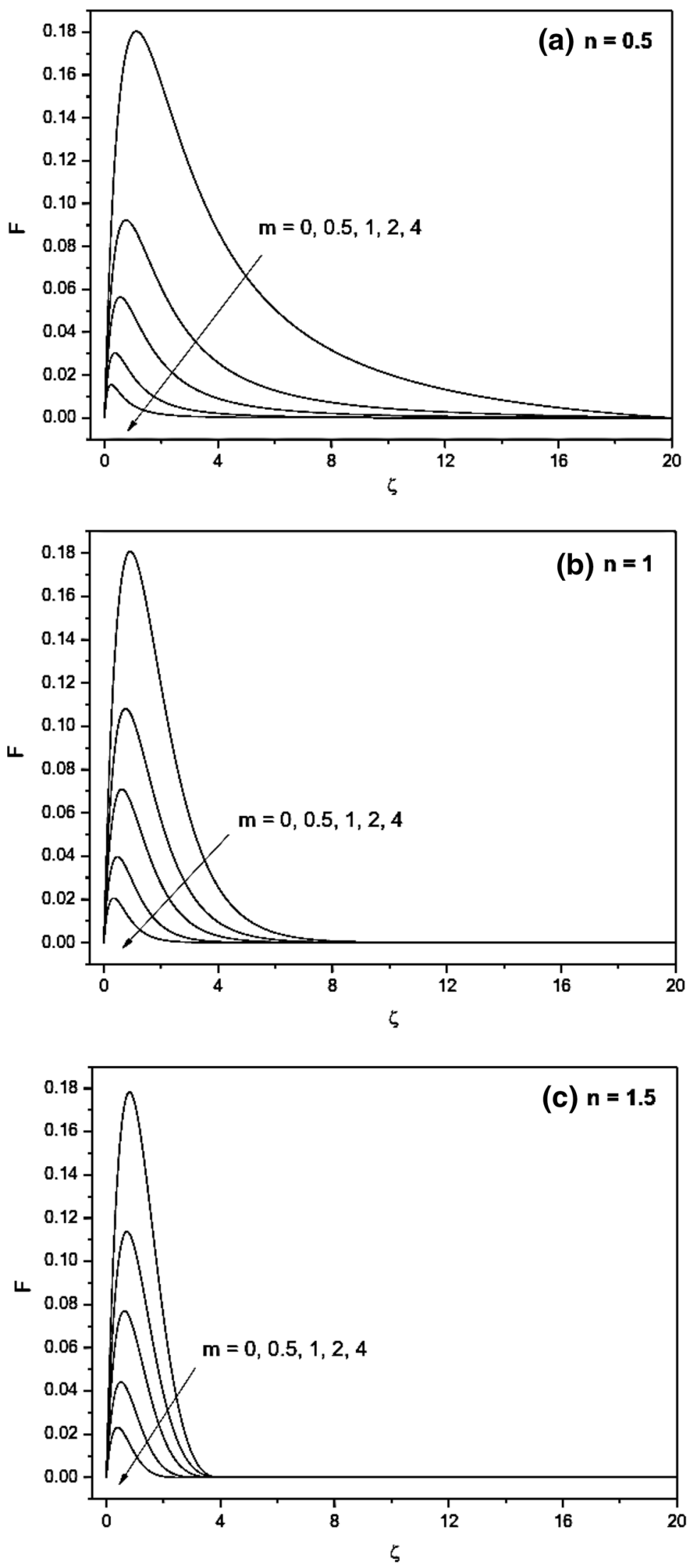
Fig. 3 Variation of the tangential velocity $G$ with different values of $m$ and $n$
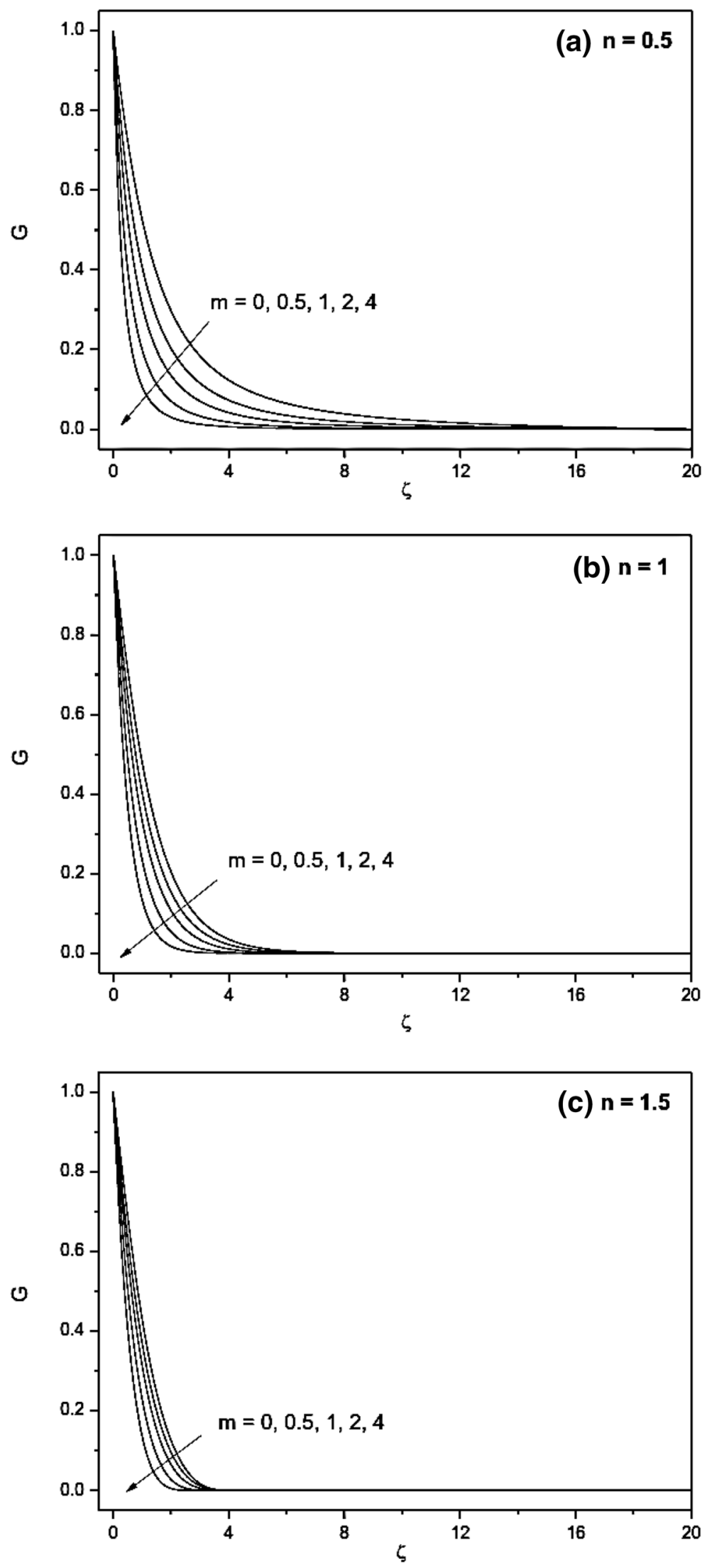
Fig. 4 Variation of the axial velocity $H$ with different values of $m$ and $n$
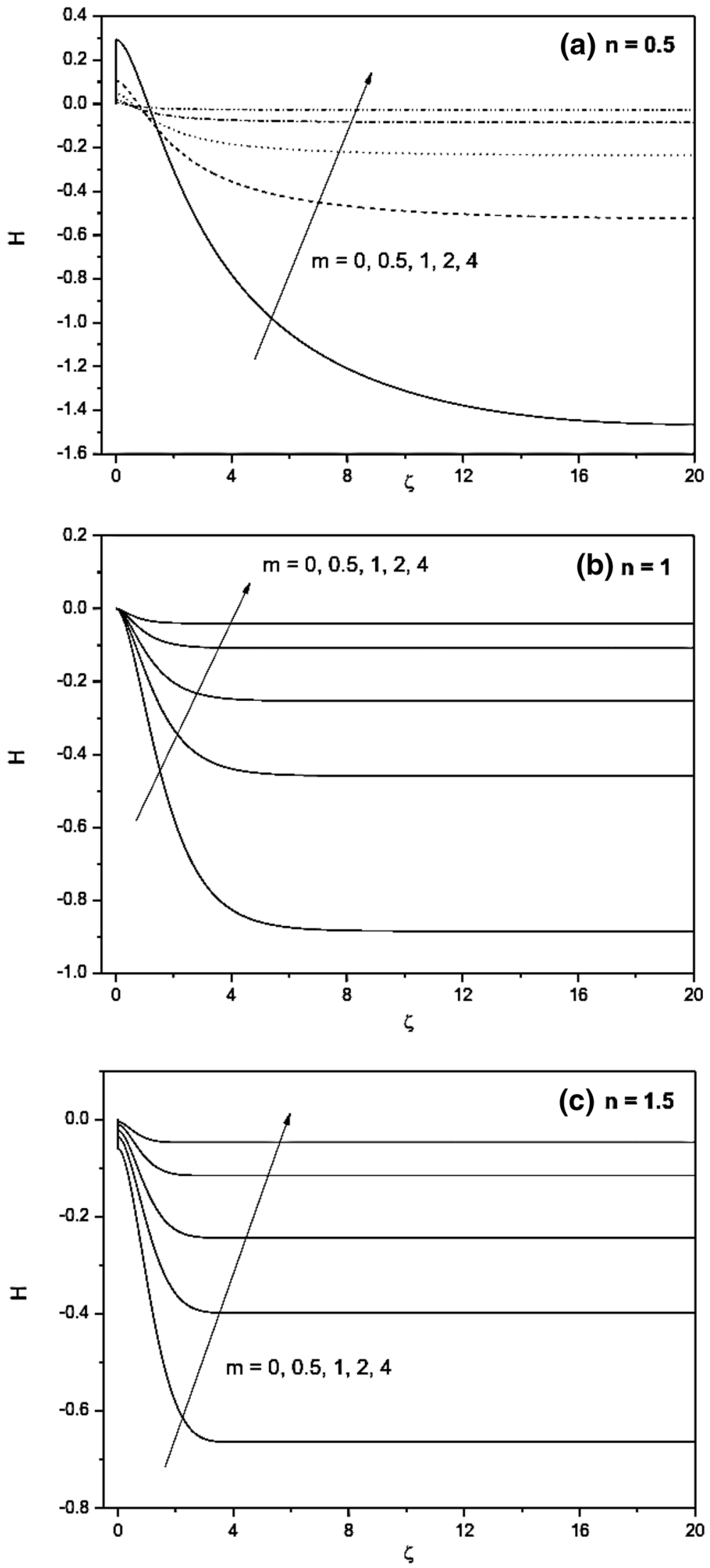
Fig. 5 Variation of both $\theta(\zeta)$ and $\phi(\zeta)$ with different values of $m$ and $n(E c=0.2$, $R_{d}=\operatorname{Pr}=L e=1, N_{t}=N_{b}=0.5$ )
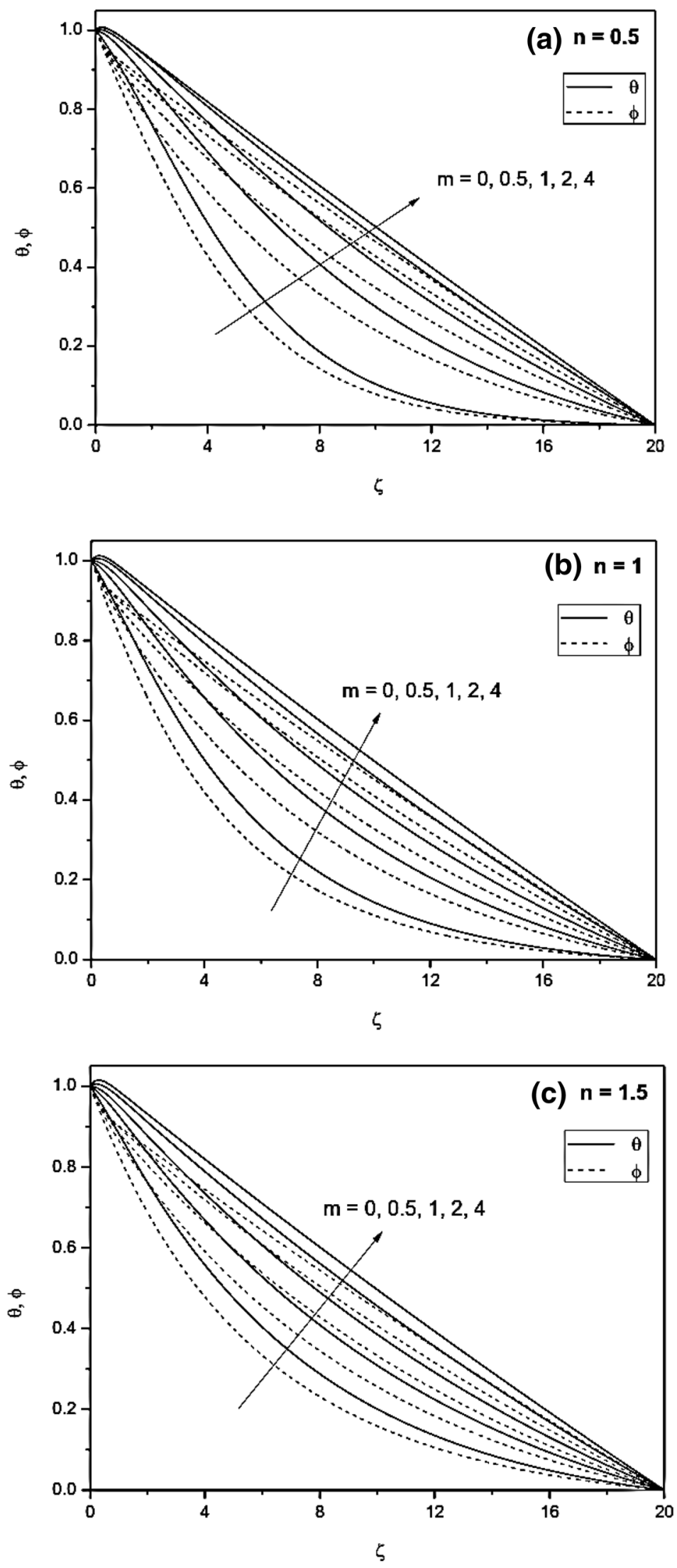
Fig. 6 Variation of both $\theta(\zeta)$ and $\phi(\zeta)$ with different values of $E c$ and $n$ ( $m=R_{d}=\operatorname{Pr}=L e=1$, $\left.N_{t}=N_{b}=0.5\right)$
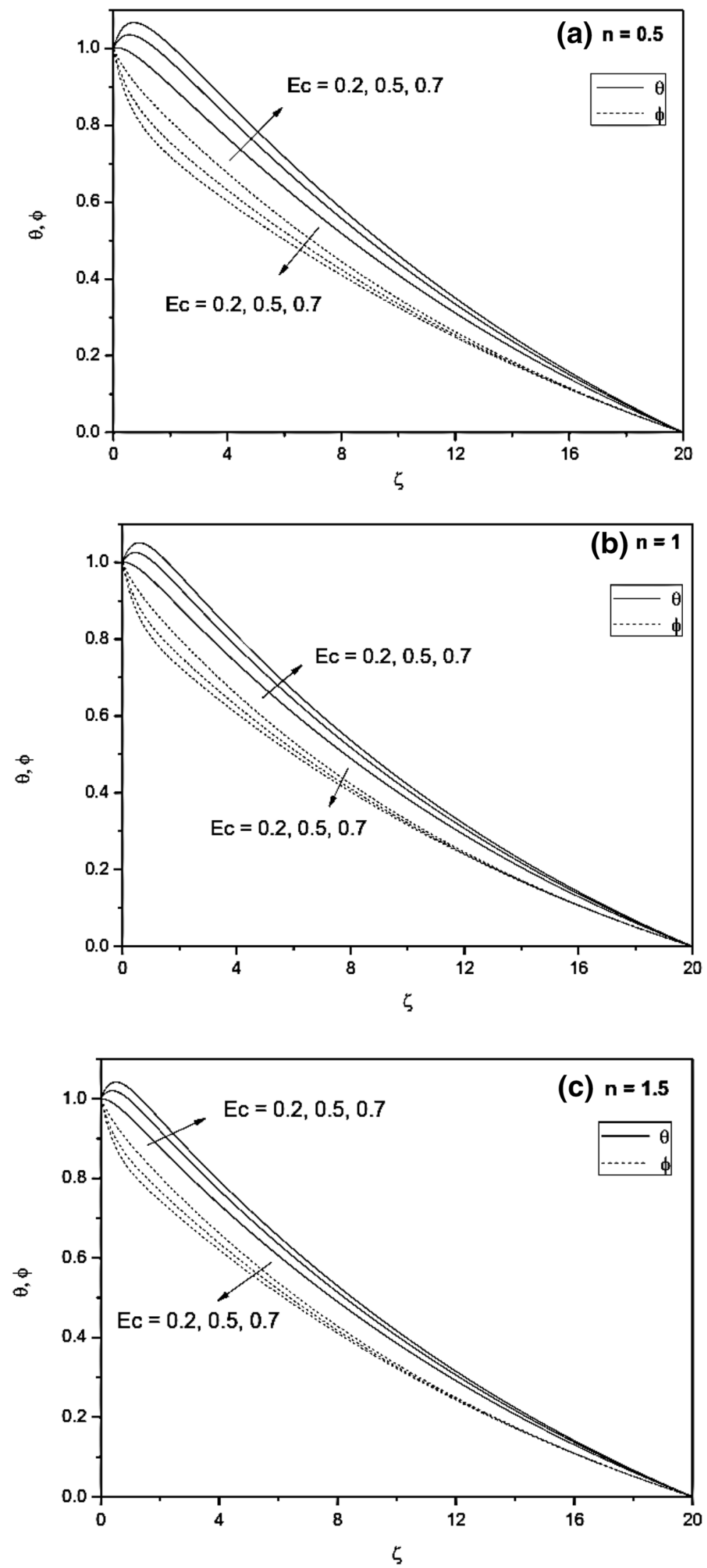
Fig. 7 Variation of both $\theta(\zeta)$ and $\phi(\zeta)$ with different values of $R_{d}$ and $n(E C=0.2$, $m=\operatorname{Pr}=L e=1, N_{t}=N_{b}=0.5$ )
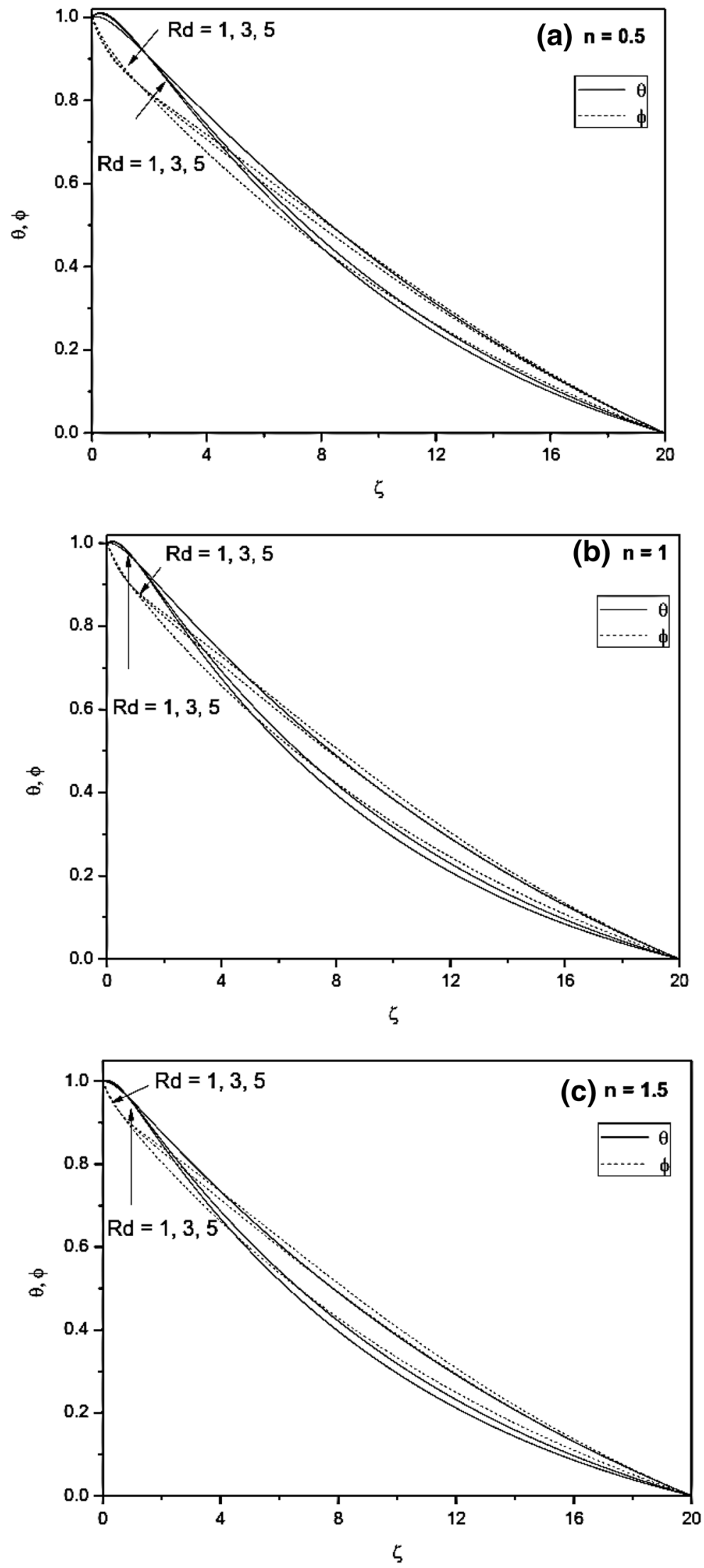
Fig. 8 Variation of both $\theta(\zeta)$ and $\phi(\zeta)$ with different values of $\operatorname{Pr}$ and $n$ (EC $=0.2$,

$R_{d}=m=L e=1, N_{t}=N_{b}=0.5$ )
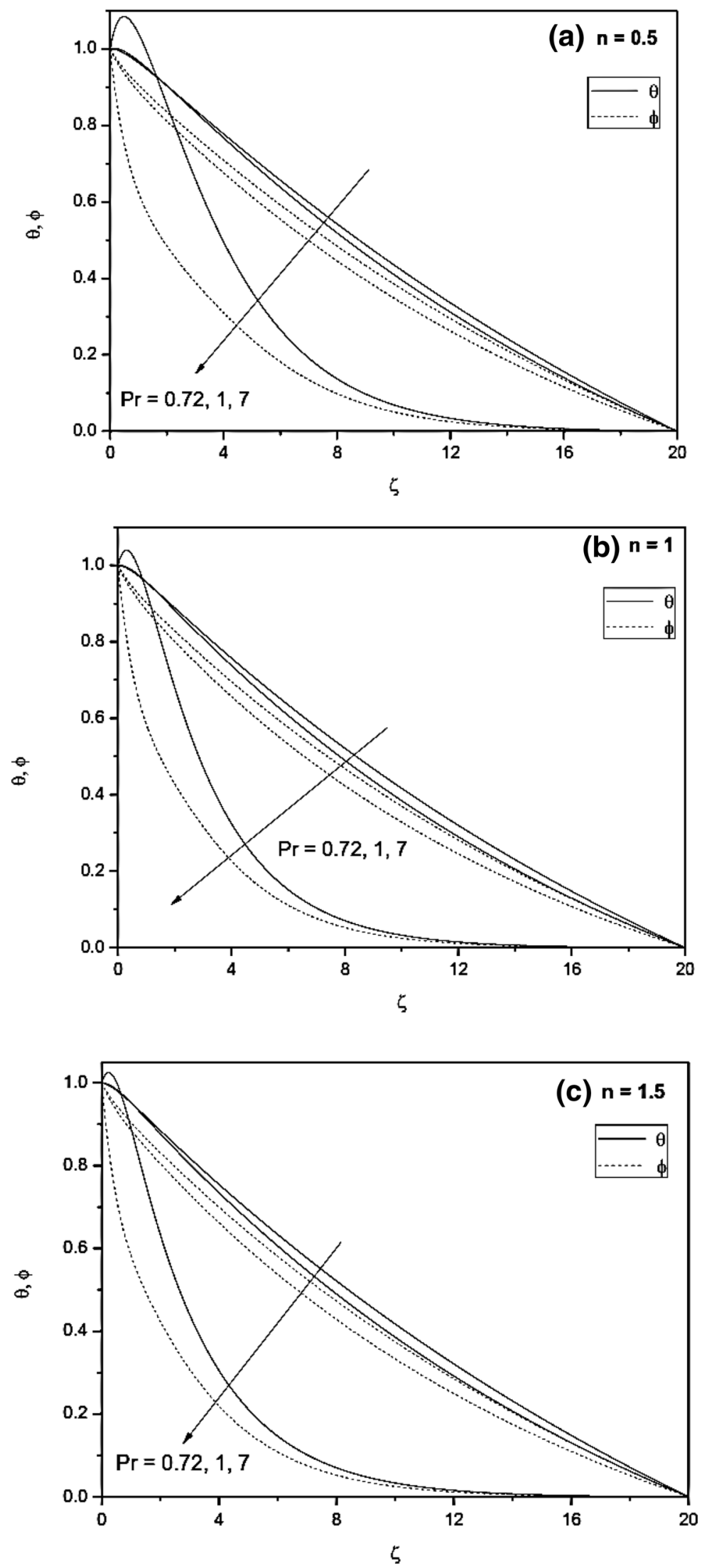
Fig. 9 Variation of both $\theta(\zeta)$ and $\phi(\zeta)$ with different values of $L e$ and $n(E C=0.2$ $R_{d}=\operatorname{Pr}=m=1, N_{t}=N_{b}=0.5$ )
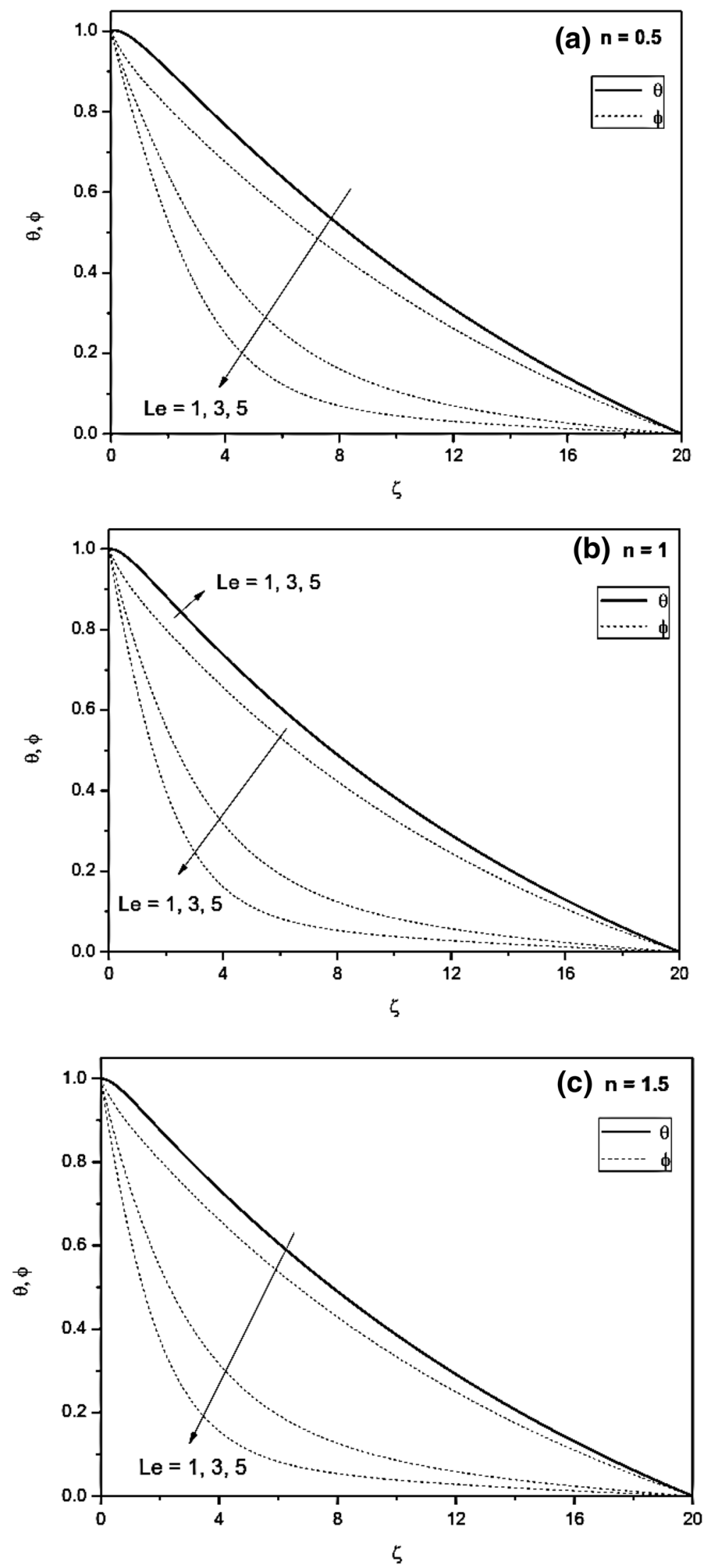
Fig. 10 Variation of both $\theta(\zeta)$ and $\phi(\zeta)$ with different values of $N_{t}$ and $n(E C=0.2$ $m=R_{d}=\operatorname{Pr}=L e=1, N_{b}=0.5$ )
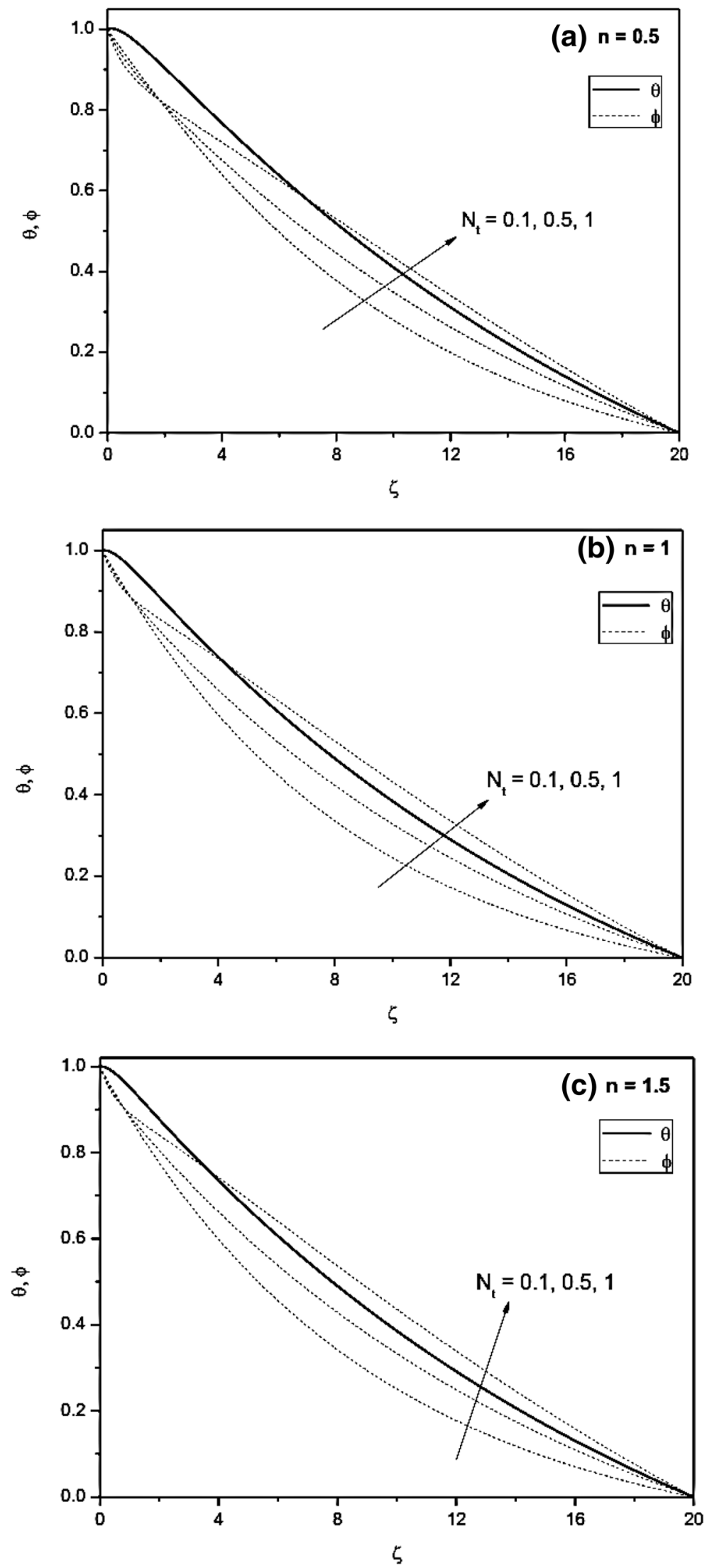
Fig. 11 Variation of both $\theta(\zeta)$ and $\phi(\zeta)$ with different values of $N_{b}$ and $n(E C=0.2$, $m=R_{d}=\operatorname{Pr}=L e=1, N_{t}=0.5$ )
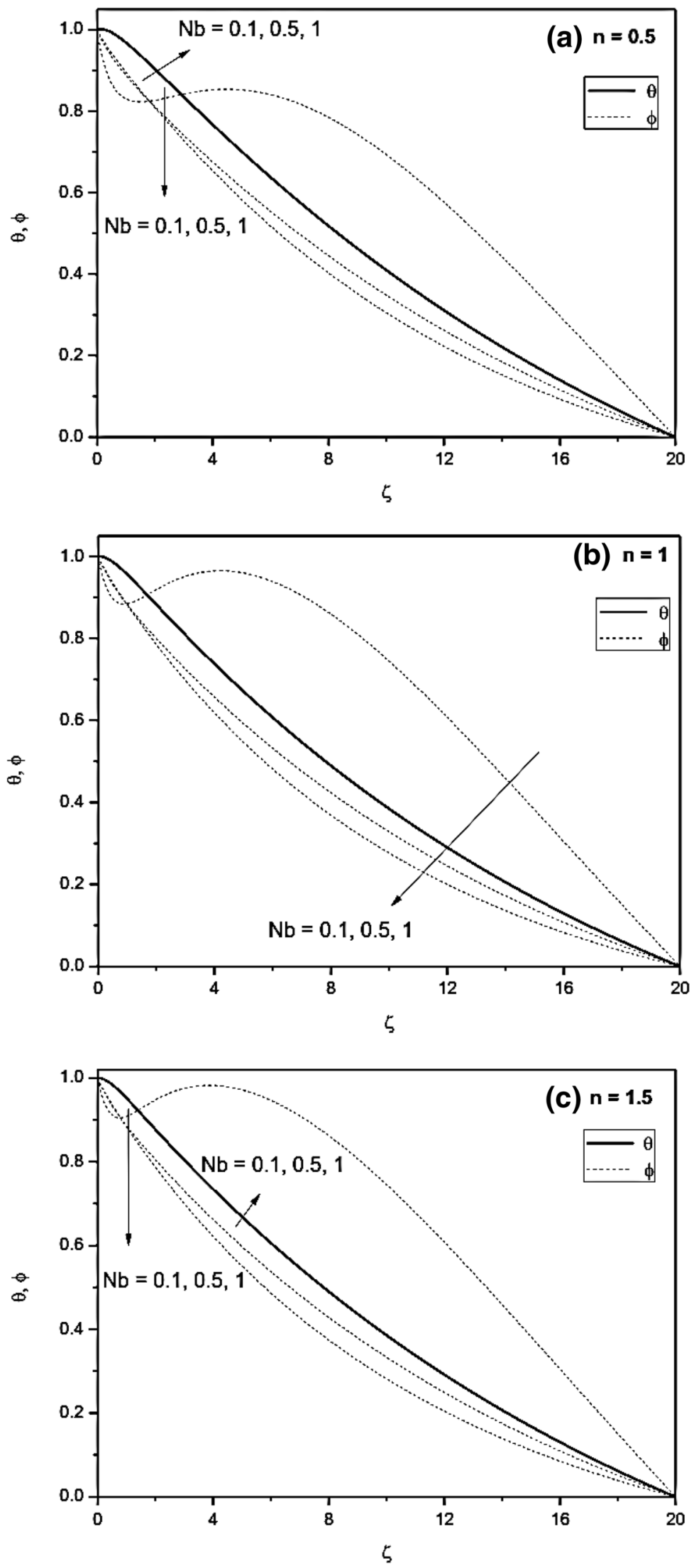


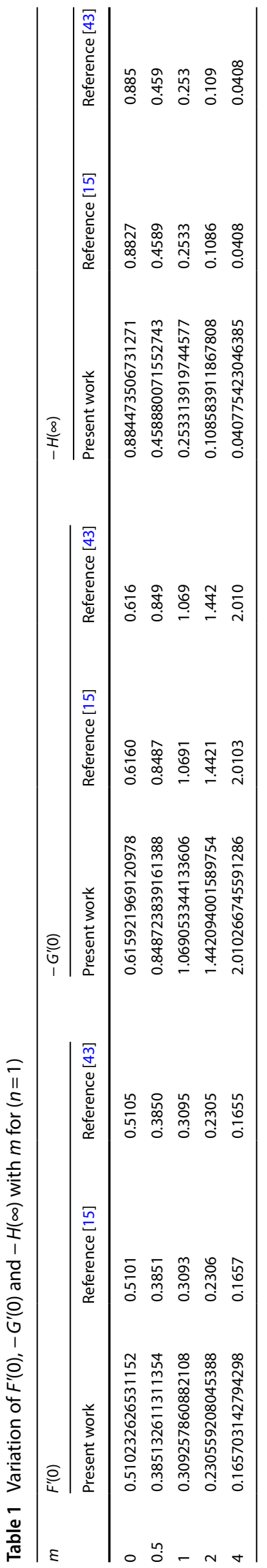

to the growth of $m$; while an increase in $-G^{\prime}(0)$ is obtained. Tables 2, 3 and 4 clarify the variation of both the radial and tangential skin frictions $F^{\prime}(0),-G^{\prime}(0)$ as well as the axial inflow $-H(\infty)$ with varying $n$. The calculations agree with those presented in $[14,20,22]$ for the non-magnetic flow $(m=0)$. It is shown that the values of the wall gradient $F^{\prime}(0)$ increase with increasing $n$, while a diminishment attitudes are obtained for both $-G^{\prime}(0)$ and $-H(\infty)$. Table 5 indicates the effectiveness of thermal radiation on the heat and mass transfer for different values of $\operatorname{Pr}$ considering a magnetic Newtonian fluid flow with $n=m=L e=1, E c=0.2$ and $N_{t}=N_{b}=0.5$. Increasing $R_{d}$ and $\operatorname{Pr}$ has a marked influence in enhancing the magnitudes of both the Nusselt and Sherwood numbers.

Table 6 elucidates the variations of the radial and tangential skin frictions, in addition to the Nusselt and Sherwood numbers for different $m$ considering different types of fluids with $\operatorname{Pr}=R_{d}=L e=1, E c=0.2$ and $N_{t}=N_{b}=0.5$. It is obvious that the radial skin friction coefficient decrease with increasing $m$ for all $n$ values, while, a raise in the magnitude of the tangential skin friction coefficient is recognized with increasing $m$ and decreasing $n$. Also, increasing $n$, enhances the value of the radial skin friction coefficient for $(m>0)$ and reduces it in the nonmagnetic flow case $(m=0)$. It is observed that for different kind of fluids, the magnitudes of both the local Nusselt number and the local Sherwood number increase with increasing the magnetic parameter $(m>1)$, while a reduction is obtained with increasing $m$, where $(m<1)$. Table 7 accentuates the variation of $S h_{r}$ for different values of $L e, N_{t}$ and $N_{b}$ where, $m=\operatorname{Pr}=R_{d}=1$ and $E c=0.2$. It is shown that $S h_{r}$ increases with increasing the parameters $L e, N_{t}$ and $N_{b}$ which accompany the flow of a nanofluid with simultaneous heat and mass transfer.

\section{Conclusions}

This research work deals with the analysis of a steady MHD flow of a non-Newtonian power law nanofluid due to the rotation of an infinite disk. The effect of thermal radiation has been enrolled together with both the Brownian motion and thermophoretic diffusion phenomena. It is concluded that increasing the magnetic parameter reduces the radial, tangential and axial velocities for all $n$ values and that the boundary layer thickness becomes thinner with increasing $n$. The augmentation of $m$ causes the boundary layer to be thinner; and the velocities variation with $m$ is more pronounced in case of non-Newtonian shear-thinning fluids. Moreover, $\theta(\zeta)$ and $\phi(\zeta)$ increase with boosting $m$ and $N_{t}$ for different $n$ values. Increasing $\operatorname{Pr}$ and $R_{d}$ decreases $\theta(\zeta)$ and the thickness of the thermal 
Table 2 Variation of $F^{\prime}(0)$ with $n$ for $(m=0)$
Table 3 Variation of $-G^{\prime}(0)$ with $n$ for $(m=0)$

\begin{tabular}{lllll}
\hline$n$ & Present work & Reference [20] & Reference [22] & Reference [14] \\
\hline 0.5 & 0.493192308796122 & 0.50058 & 0.501 & 0.501 \\
0.6 & 0.49759630965858 & - & 0.501 & 0.500 \\
0.8 & 0.504218392738096 & 0.50381 & 0.504 & 0.504 \\
0.9 & 0.507189214573548 & - & 0.507 & 0.507 \\
1 & 0.510232626531152 & 0.51021 & 0.510 & 0.510 \\
1.1 & 0.51338911702259 & - & 0.514 & 0.514 \\
1.3 & 0.519894465021008 & 0.52150 & 0.522 & 0.521 \\
1.5 & 0.526405459661366 & 0.52919 & 0.529 & 0.529 \\
\hline
\end{tabular}

\begin{tabular}{lllll}
\hline$n$ & Present work & Reference [20] & Reference [22] & Reference [14] \\
\hline 0.5 & 0.63258191586443 & 0.71322 & 0.712 & 0.713 \\
0.6 & 0.62813774055592 & - & 0.676 & 0.677 \\
0.8 & 0.621449576816618 & 0.63608 & 0.636 & 0.636 \\
0.9 & 0.618427949901908 & - & 0.624 & 0.624 \\
1 & 0.615921969120978 & 0.61591 & 0.616 & 0.616 \\
1.1 & 0.614173278357886 & - & 0.610 & 0.610 \\
1.3 & 0.612860027915832 & 0.60346 & 0.603 & 0.603 \\
1.5 & 0.613704176064384 & 0.60099 & 0.601 & 0.601 \\
\hline
\end{tabular}

Table 4 Variation of $-H(\infty)$ with $n$ for $(m=0)$

\begin{tabular}{lllll}
\hline$n$ & Present work & Reference [20] & Reference [22] & Reference [14] \\
\hline 0.5 & 1.46460907950617 & 1.54389 & 1.539 & 1.513 \\
0.6 & 1.36013776301004 & - & 1.364 & 1.351 \\
0.8 & 1.09399919164270 & 1.05929 & 1.089 & 1.052 \\
0.9 & 0.975412633859303 & - & 0.969 & 0.958 \\
1 & 0.884473506731271 & 0.88230 & 0.883 & - \\
1.1 & 0.817372662809852 & - & 0.822 & 0.819 \\
1.3 & 0.724913567797793 & 0.73591 & 0.735 & 0.735 \\
1.5 & 0.663515470870505 & 0.67828 & 0.676 & 0.678 \\
\hline
\end{tabular}

boundary layer, while, increasing Ec enhances the temperature values.

Furthermore, the temperature of the fluid decreases with increasing $N_{b}$ for Newtonian fluids; while, an elevation in $\theta(\zeta)$ profiles is obtained with increasing $N_{b}$ regarding the class of non-Newtonian fluids $(n \neq 1)$, which totally opposes the effect of $L e$ on $\theta(\zeta)$. On the other hand, a diminishment attitude of $\phi(\zeta)$ is obtained due to the increase of $E c, \operatorname{Pr}$, Le and $N_{b}$, while, an elevation of the behavior of $\phi(\zeta)$ is observed with increasing $R_{d}$ for different $n$ values. The wall gradient $F^{\prime}(0)$ and the axial inflow $-H(\infty)$ decrease; while, the values of $-G^{\prime}(0)$ increase with increasing $m$. The values of $F^{\prime}(0)$ increase, while, a diminishment attitudes are obtained for both $-G^{\prime}(0)$ and $-H(\infty)$ with increasing $n$. Increasing $R_{d}$ and $P r$ has a marked influence in enhancing the magnitudes of both $N u_{r}$ and $S h_{r}$. The radial skin friction coefficient decreases with increasing $m$ for all $n$ values, while, the magnitude of the tangential skin friction coefficient raises with increasing $m$ and decreasing $n$. Also, increasing $n$ enhances the radial skin friction coefficient for $(m>0)$ and reduces it in the nonmagnetic flow case $(m=0)$. The magnitudes of both $\mathrm{Nu}_{r}$ and $\mathrm{Sh}_{r}$ increase with increasing the magnetic parameter $(m>1)$, while a reduction is obtained with increasing $m$, where $(m<1)$. Moreover, increasing the parameters $L e, N_{t}$ and $N_{b}$ put up the values of $S h_{r}$. 
Table 5 Variation of $\mathrm{Nu}_{r}$ and $\mathrm{Sh}_{r}$ with $R_{d}$ and $\mathrm{Pr}$

\begin{tabular}{|c|c|c|c|c|c|c|}
\hline \multirow[t]{2}{*}{$R_{d}$} & \multicolumn{2}{|l|}{$\operatorname{Pr}=0.72$} & \multicolumn{2}{|l|}{$P r=1$} & \multicolumn{2}{|l|}{$\operatorname{Pr}=7$} \\
\hline & $\operatorname{Re}_{r}^{\frac{-1}{1+n}} S h_{r}$ & $\operatorname{Re}_{r}^{\frac{-1}{1+n}} N u_{r}$ & $\operatorname{Re}_{r}^{\frac{-1}{1+n}} S h_{r}$ & $\operatorname{Re}_{r}^{\frac{-1}{1+n}} N u_{r}$ & $\operatorname{Re}_{r}^{\frac{-1}{1+n}} S h_{r}$ & $\operatorname{Re}_{r}^{\frac{-1}{1+n}} N u_{r}$ \\
\hline 1 & 0.0166894795654587 & 0.156252526581046 & -0.0198871646156568 & 0.170140512089961 & -0.688245254572995 & 0.445089021084561 \\
\hline 3 & -0.0251662505889639 & 0.178063581296468 & -0.0596735306589791 & 0.199504596559579 & -0.725631591269424 & 0.654167626988621 \\
\hline 5 & -0.0331528921745256 & 0.185885838639042 & -0.0670057362119886 & 0.210010132113324 & -0.735161095475417 & 0.732829155305063 \\
\hline 10 & -0.0389858663613412 & 0.193271400407583 & -0.0722702067891076 & 0.219935609173871 & -0.742845974805062 & 0.808431333904430 \\
\hline $10^{9}$ & -0.0446388814336086 & 0.202512925429210 & -0.0772831874432836 & 0.232380241876180 & -0.750974909816036 & 0.904544099433605 \\
\hline
\end{tabular}

Table 6 Variation of $C_{f_{t}}, C_{f_{r}}, N u_{r}$ and $S h_{r}$ for various values of $n$ and $m$

\begin{tabular}{cllll}
\hline$m$ & $\mathrm{Re}_{r}^{\frac{1}{1+n}} C_{f_{r}}$ & $\mathrm{Re}_{r}^{\frac{1}{1+n}} C_{f_{t}}$ & $\mathrm{Re}_{r}^{\frac{-1}{1+n}} N u_{r}$ & $\mathrm{Re}_{r}^{\frac{-1}{1+n}} S h_{r}$ \\
\hline (a) $n=0.5$ & & & \\
0 & 0.550676164119244 & -0.706312277597639 & 0.201638342300127 & 0.176522064545761 \\
0.5 & 0.365795959526631 & -0.970309343075206 & 0.0325647742432875 & 0.169194598108047 \\
1 & 0.265782152935280 & -1.16889249624685 & -0.0606377282971172 & 0.168153870538071 \\
2 & 0.176585514326603 & -1.45045675387478 & -0.149060220418004 & 0.180418763015762 \\
4 & 0.112964357780706 & -1.81975871983747 & -0.236544166196381 & 0.207176542227096 \\
(b) $n=1$ & & & \\
0 & 0.510232626531151 & -0.615921969120977 & 0.262730206632899 & 0.206801680240763 \\
0.5 & 0.385132611311354 & -0.848723839161388 & 0.0864261226819529 & 0.177338284204363 \\
1 & 0.309257860882109 & -1.06905334413361 & -0.0198871646156568 & 0.170140512089961 \\
2 & 0.230559208045388 & -1.44209400158975 & -0.136506973946131 & 0.184067627834636 \\
4 & 0.165703142794299 & -2.01026674559129 & -0.269087059349429 & 0.224797685689891 \\
(c) $n=1.5$ & & & \\
0 & 0.473337492951759 & -0.551835454554766 & 0.239167297720755 & 0.191678236344924 \\
0.5 & 0.376179073206399 & -0.771060242685149 & 0.0952451340594148 & 0.169530102672334 \\
1 & 0.315896168904793 & -0.993362081912373 & -0.00261752400267962 & 0.165167760664571 \\
2 & 0.250076649975373 & -1.40160263844908 & -0.124817407951439 & 0.182126293719031 \\
4 & 0.192121377372538 & -2.07643792230088 & -0.279628936553652 & 0.231126183033520 \\
\hline
\end{tabular}

Table 7 Variation of $S h_{r}$ with different values of $L e, N_{t}$ and $N_{b}$

\begin{tabular}{lllll}
\hline $\mathrm{Re}_{r}^{\frac{-1}{1+n}} S h_{r}$ & $N_{t}$ & $N_{b}=1$ & $N_{b}=0.5$ & $N_{b}=0.1$ \\
\hline (a) $L e=1$ & 0.1 & 0.123727433153968 & 0.128884441924629 & 0.170140512089961 \\
& 0.5 & 0.144355468236633 & 0.170140512089961 & 0.376420862916632 \\
& 1 & 0.170140512089961 & 0.221710599796625 & 0.634271301449964 \\
(b) $L e=3$ & 0.1 & 0.266522896495730 & 0.271296882852467 & 0.309488773706371 \\
& 0.5 & 0.285618841922669 & 0.309488773706371 & 0.500448227975631 \\
& 1 & 0.309488773706371 & 0.357228637273763 & 0.739147545812722 \\
(c) $L e=5$ & 0.1 & 0.375089927738802 & 0.379884608872757 & 0.418242057944828 \\
& 0.5 & 0.394268652274897 & 0.418242057944828 & 0.610029303297981 \\
& 1 & 0.418242057944828 & 0.466188869285131 & 0.849763359998881 \\
\hline
\end{tabular}

\section{Compliance with ethical standards}

Conflict of interest The authors declare that they have no conflict of interest.

\section{References}

1. Attia HA, Essawy MAI (2016) Numerical analysis for a parametric study of a steady non-Darcian flow over a rotating disk in a porous medium. Int J Basic Sci Appl Res 5(1):1-12 
2. Kármán TV (1921) Über laminare und turbulente Reibung. Z Angew Math Mech 1:233-252. https://doi.org/10.1002/ zamm.19210010401

3. Millsaps K, Pohlhausen K (1952) Heat transfer by laminar flow from a rotating plate. J Aeronaut Sci 19:120-125

4. Sparrow EM, Gregg JL (1960) Mass transfer, flow and heat transfer about a rotating disk. ASME J Heat Transf 82:294-302

5. Attia HA (1998) Unsteady MHD flow near a rotating porous disk with uniform suction or injection. Fluid Dyn Res 23(5):283-290

6. Batista M (2011) Steady flow of incompressible fluid between two co-rotating disks. Appl Math Model 35(10):5225-5233

7. Chamekh M, Elzaki TM (2018) Explicit solution for some generalized fluids in laminar flow with slip boundary conditions. J Math Comput Sci 18:272-281

8. Griffiths PT (2015) Flow of a generalised Newtonian fluid due to a rotating disk. J Nonnewton Fluid Mech 221:9-17

9. Chamekh M, Brik N, Abid M (2018) Mathematical and Numerical study of the concentration effect of red cells in blood. J King Saud Univ Sci. https://doi.org/10.1016/j.jksus.2018.11.004

10. Andersson HI, Bech KH, Dandapat BS (1992) Magnetohydrodynamic flow of a power-law fluid over a stretching sheet. Int $J$ Non-Linear Mech 27(6):929-936

11. Djukic DS (1973) On the use of Croccos equation for the flow of power-law fluids in a transverse magnetic field. AIChE J 19:1159-1163

12. Djukic DS (1974) Hiemenz magnetic flow of power-law fluids. Trans ASME J Appl Mech 41:822-823

13. Mitschka P (1964) Nicht-Newtonsche flüssigkeiten II. Drehströmungen Ostwald-de Waelescher nicht-Newtonscher flüssigkeiten. Collect Czechoslov Chem Commun 29:2892-2905

14. Mitschka P, Ulbreche J (1965) Nicht-Newtonsche flüssigkeiten IV. Strömung nicht-Newtonscher flüssigkeiten Ostwald-deWaeleschen typs in der umgebung rotierender drehkegel und scheiben. Collect Czechoslov Chem Commun 30:2511-2526

15. Andersson $\mathrm{HI}$, de Korte E (2002) MHD flow of a power-law fluid over a rotating disk. Eur J Mech B Fluids 21:317-324

16. Attia HA, Ewis KM, Abd-maksoud IH, Abdeen MAM (2012) Steady hydromagnetic flow of a non-Newtonian power law fluid due to a rotating porous disk with heat transfer. Russ J Phys Chem A 86(13):2063-2070

17. Attia HA, Ewis KM, Abd-elmaksoud IH, AwadAllah NA (2012) Hydromagnetic rotating disk flow of a non-Newtonian fluid with heat transfer and Ohmic heating. KSIAM J 16(3):169-180

18. Bachok N, Ishak A, Pop I (2011) Flow and heat transfer over a rotating porous disk in a nanofluid. Phys B 406:1767-1772

19. Anjali Devi SP, Devi RU (2011) Soret and Dufour effects on MHD slip flow with thermal radiation over a porous rotating infinite disk. Commun Nonlinear Sci Numer Simul 16(4):1917-1930

20. Ming $C$, Zheng L, Zhang $X$ (2011) Steady flow and heat transfer of the power-law fluid over a rotating disk. Int Commun Heat Mass Transf 38:280-284

21. Osalusi E, Side J, Harris R, Johnston B (2007) On the effectiveness of viscous dissipation and Joule heating on steady MHD flow and heat transfer of a Bingham fluid over a porous rotating disk in the presence of Hall and ion-slip currents. Int Commun Heat Mass Transf 34:1030-1040

22. Andersson HI, de Korte E, Meland R (2001) Flow of a power-law uid over a rotating disk revisited. Fluid Dyn Res 28:75-88

23. Choi SUS (1995) Enhancing thermal conductivity of fluids with nanoparticle. In: Siginer DA, Wang HP (eds) Developments and applications of non-Newtonian flows, vol FED 231. ASME, New York, pp 99-105
24. Buongiorno J (2006) Convective transport in nanofluids. J Heat Transf 128:240-250

25. Masuda H, Ebata A, Teramae K, Hishinuma N (1993) Alteration of thermal conductivity and viscosity of liquid by dispersed ultra-fine particles (dispersion of $\mathrm{Al}_{2} \mathrm{O}_{3}, \mathrm{SiO}_{2}$, and $\mathrm{TiO}_{2}$ ultra-fine particles). NetsuBussei 4:227-233

26. Buongiorno J, Hu W (2005), Nanofluid coolants for advanced nuclear power plants. In: Proceedings of international congress on advances in nuclear power plants, Carran Associate, Inc., 5705, Seoul, 2005

27. Prabhat N, Buongiorno J, Hu L-W (2012) Convective heat transfer enhancement in nanofluids: real anomaly or analysis artifact? J Nanofluids 1(1):55-62

28. Rashidi MM, Abelman S, Freidooni Mehr N (2013) Entropy generation in steady MHD flow due to a rotating porous disk in a nanofluid. Int J Heat Mass Transf 62:515-525

29. Turkyilmazoglu M (2014) Nanofluid flow and heat transfer due to a rotating disk. Comput Fluids 94:139-146

30. Li B, Chen X, Zheng L, Zhu L, Zhou J, Wang T (2014) Precipitation phenomenon of nanoparticles in power-law fluids over a rotating disk. Microfluid Nanofluid 17:107-114

31. Mustafa M, Khan JA, Hayat T, Alsaedi A (2015) On Bödewadt flow and heat transfer of nanofluids over a stretching stationary disk. J Mol Liq 211:119-125

32. Imtiaz M, Hayat T, Alsaedi A, Ahmad B (2016) Convective flow of carbon nanotubes between rotating stretchable disks with thermal radiation effects. Int J Heat Mass Transf 101:948-957

33. Hatami M, Sheikholeslami M, Ganji DD (2014) Laminar flow and heat transfer of nanofluid between contracting and rotating disks by least square method. Powder Technol 253:769-779

34. Hayat T, Qayyum S, Imtiaz M, Alzahrani F, Alsaedi A (2016) Partial slip effect in flow of magnetite- $\mathrm{Fe}_{3} \mathrm{O}_{4}$ nanoparticles between rotating stretchable disks. J Magn Magn Mater 413:39-48

35. Saidi MH, Tamim H (2016) Heat transfer and pressure drop characteristics of nanofluid in unsteady squeezing flow between rotating porous disks considering the effects of thermophoresis and Brownian motion. Adv Powder Technol 27:564-574

36. EL-Dabe NT, Attia HA, Essawy MAl, Ramadan AA, Abdel-Hamid $\mathrm{AH}$ (2016) Non-linear heat and mass transfer in a MHD Homann nanofluid flow through a porous medium with chemical reaction, heat generation and uniform inflow. Eur Phys J Plus 131(11):395. https://doi.org/10.1140/epjp/i2016-16395-8

37. Necati Özisik M (1973) Radiative transfer. Wiley, New York

38. Anjali Devi SP, Uma Devi R (2011) On hydromagnetic flow due to a rotating disk with radiation effects. Nonlinear Anal Model Control 16(1):17-29

39. Ames WF (1977) Numerical solutions of partial differential equations, 2nd edn. Academic Press, New York

40. Mitchell AR, Griffiths DF (1980) The finite difference method in partial differential equations. Wiley, New York

41. Evans GA, Blackledge JM, Yardley PD (2000) Numerical methods for partial differential equations. Springer, New York

42. Hashmi MM, Hayat T, Alsaedi A (2012) On the analytic solutions for squeezing flow of nanofluid between parallel disks. Nonlinear Anal Model Control 17:418-430

43. Sparrow EM, Cess RD (1962) Magnetohydrodynamic flow and heat transfer about a rotating disk. ASME J Appl Mech 29:181-187

Publisher's Note Springer Nature remains neutral with regard to jurisdictional claims in published maps and institutional affiliations. 